\title{
Clinicopathological alterations in naturally occurring Babesia gibsoni infection in dogs of Middle-South Gujarat, India
}

\author{
Avinash K. Bilwal, Ghanshyam C. Mandali and Falguni B. Tandel \\ Department of Veterinary Medicine, College of Veterinary Science and Animal Husbandry, Anand Agricultural University, \\ Anand, Gujarat, India. \\ Corresponding author: Avinash K. Bilwal, e-mail: akbilwal.vet@gmail.com \\ Co-authors: GCM: gcmandali@aau.in, FBT: tandelfalguni1890@gmail.com \\ Received: 24-05-2017, Accepted: 14-09-2017, Published online: 14-10-2017
}

doi: 10.14202/vetworld.2017.1227-1232 How to citethis article: Bilwal AK, Mandali GC, Tandel FB(2017)Clinicopathological alterations in naturally occurring Babesia gibsoni infection in dogs of Middle-South Gujarat, India, Veterinary World, 10(10): 1227-1232.

\begin{abstract}
Aim: The present research work was undertaken to describe various clinical signs and hematobiochemical alterations in dogs affected with Babesia gibsoni.

Materials and Methods: Blood smears from a total of 79 suspected dogs of Anand region as well as Surat region of Gujarat state (India) were screened for detection of intraerythrocytic piroplasm of small form of Babesia. Diagnosis was made on the basis of clinical signs and demonstration of B. gibsoni organism in Giemsa-stained thin blood smears. The clinical signs were recorded at the time of presentation, and blood samples were subjected to estimation of hematobiochemical parameters by auto hematology analyzers at College of Veterinary Science and Animal Husbandry, Anand. Statistical analysis, interpretation, and comparison of hematobiochemical changes with scientific literature were carried out to understand the pathophysiology of the disease.

Results: Out of 79 dogs, 16 were positive for naturally occurring babesiosis based on the presence of intraerythrocytic piroplasm of small form of Babesia in blood smears. The clinical cases were manifested by wide variety of non-specific clinical signs. The hematological evaluation revealed that the mean values of hemoglobin and total erythrocyte counts in dogs with babesiosis decreased significantly $(\mathrm{p}<0.01)$ in comparison to healthy dogs. Among differential leukocyte count, mean values of neutrophils and eosinophils increased while lymphocytes decreased $(\mathrm{p}<0.01)$ in dogs with babesiosis in comparison to healthy dogs. Serum biochemistry revealed increase $(p<0.01)$ value of alkaline phosphatase, alanine aminotransferase, aspartate aminotransferase, and globulin as well as decrease in albumin levels $(p<0.05)$ in dogs with babesiosis as compared to healthy dogs.
\end{abstract}

Conclusion: B. gibsoni is having multisystemic effects with atypical hematobiochemical changes in dog are discussed here, which would aid new insights in diagnosis of disease.

Keywords: babesiosis, clinicopathological changes, dogs, intraerythrocytic piroplasm.

\section{Introduction}

Blood-feeding ectoparasites such as ticks, fleas, sand flies, and mosquitoes can transmit many dangerous pathogens to dogs - such as bacteria, protozoa, viruses, or helminths. They may lead to a variety of serious infections, mostly classified by their vectors: Tick-borne diseases, flea-borne diseases, sand fly-borne diseases, and mosquito-borne diseases [1]. Tick-borne hemoparasites are one of the most important vector-borne infections of dogs. They are numerous and are caused by several etiological agents such as bacterial, protozoan, and rickettsial organisms [2]. Among them, tick-borne hemopathogens such as Babesia, Ehrlichia, Anaplasma, Borrelia, and Hepatozoon are of major health concern to dogs and some of which are of zoonotic significance

Copyright: Bilwal, et al. Open Access. This article is distributed under the terms of the Creative Commons Attribution 4.0 International License (http://creativecommons.org/licenses/by/4.0/), which permits unrestricted use, distribution, and reproduction in any medium, provided you give appropriate credit to the original author(s) and the source, provide a link to the Creative Commons license, and indicate if changes were made. The Creative Commons Public Domain Dedication waiver (http://creativecommons.org/ publicdomain/zero/1.0/) applies to the data made available in this article, unless otherwise stated.
[3]. Canine babesiosis is a clinically significant and geographically widespread hemoprotozoan disease of domesticated dogs and wild canids [4]. The commonly occurring Babesia species in dogs are the Babesia canis and Babesia gibsoni [5]. Based on genetic data, vector specificity and variation of pathogenicity, large Babesia are subdivided into three species, namely, B. canis transmitted by Dermacentor reticulatus (in Europe), B. vogeli transmitted by Rhipicephalus sanguineus (in tropical and subtropical regions), and $B$. rossi transmitted by Haemaphysalis elliptica (in South Africa). Small Babesia includes B. gibsoni (Asian genotype), Babesia conradae (previously Californian genotype), and Babesia microti like species (also referred to as the Spanish isolate or Theileria annae) [6]. The disease has been reported in various states of India including Gujarat [7-10]. The life cycle of B. gibsoni includes two stages: Inside the host red blood cells (RBCs), in which the sporozoites convert into piroplasms and the other inside the tick vector [11]. A small form of Babesia is seen in Giemsa-stained peripheral blood smear of small 1-3 $\mu \mathrm{m}$ in diameter, ring-, oval-, or comma-shaped piroplasms suggestive of $B$. gibsoni which can be 
confirmed by polymerase chain reaction [12]. The parasite also transmitted by blood exchange and transplacental route can also be possible [13]. The immunological response plays the most important role in pathogenesis of canine babesiosis. These parasites initiate a mechanism of antibody-mediated cytotoxic destruction of circulating erythrocytes. Autoantibodies are directed against components of the membranes of infected and uninfected erythrocytes which causes intravascular and extravascular hemolysis $[14,15]$.

The clinical-pathological changes, including hematology and blood chemistry, are nonspecific. The various clinical symptoms regularly depend on the severity of the disease in infected animals. The typical clinical findings include anemia, thrombocytopenia, leukocyte abnormalities, increased liver enzymes, and hyperbilirubinemia. hypokalemia, hyperglobulinemia, azotemia, metabolic acidosis, and abnormalities of urinalysis may be observed in some severely affected dogs [16]. Supportive treatment is usually given and includes fluid therapy, anti-inflammatory and antipyretics, gastroprotectants, oxygen supplementation, and blood transfusion should be employed when necessary.

The naturally occurring cases of $B$. gibsoni are having variety of clinical manifestations ranging from anorexia to hepatomegaly or splenomegaly or death making it difficult to have a definitive diagnosis solely on the basis of clinical examination. Hence, the study was conducted to describe various clinical signs and hematobiochemical alterations in dogs affected with B. gibsoni.

\section{Materials and Methods \\ Ethical approval}

Samples were collected from clinical cases coming to Teaching Veterinary Clinical Complex (TVCC), Veterinary College, Anand Agricultural University, Anand and Nandini Veterinary Hospital, Surat. Hence, this particular study did not require ethical approval.

\section{Sample collection}

Privately owned dogs presented to TVCC, Anand and Nandini Veterinary Hospital, Surat, Gujarat (India), were examined clinically irrespective of their age, breed, and sex from July 2015 to March 2016. Dogs showing clinical signs suggestive of babesiosis were screened out during period and among that total 79 peripheral blood smears were made from suspected dogs and examined for the presence of intraerythrocytic piroplasms. Dogs presented to clinics for checkup which are healthy, from ectoparasites dewormed, and vaccinated were used as healthy control group, and blood was collected from them aseptically. Diagnosis of babesiosis was confirmed by cytological examination of Giemsa-stained peripheral blood smears made from ear tips. A $5 \mathrm{ml}$ of blood samples from positive dogs were collected in a sterile anticoagulant vial containing ethylenediaminetetraacetic acid $\left(\mathrm{K}_{3}\right)$. Hematological parameters were analyzed by auto hematoanalyzer (Analytical, Hema 2062, Analytical Technologies Limited, Vadodara, India). Serum biochemical parameters were analyzed using an auto-biochemical analyzer (Mindray, BS-120 chemistry analyzer) using standard kits manufactured by coral clinical systems auto-chemistry analyzer using commercial diagnostic kits procured from Crest Biosystem (A Division of Coral Clinical System, Goa) at the Department of Veterinary Physiology and Biochemistry, College of Veterinary Science and Animal Husbandry, Anand with standard laboratory protocols. Hematobiochemical changes in dogs with naturally occurring babesiosis were compared with healthy dogs.

\section{Statistical analysis}

The data obtained were subjected to the statistical analysis described by Snedecor and Cochran [17]. The independent t-test having means with unequal variances was carried out. Variables with $\mathrm{p}<0.05$ were considered as statistically "significant," variables with $\mathrm{p}<0.01$ were considered as statistically "highly significant" and variables with $p>0.05$ were considered as statistically "non-significant."

\section{Results and Discussion}

About 16 out of 79 peripheral blood smears were having small single oval piroplasms inside the red blood cells indicative of $B$. gibsoni, so the overall prevalence was $20.25 \%$. Little information in literatures is available on prevalence in Gujarat. Jadhav et al. [10] studied the epidemiology of canine babesiosis in Gujarat, and that was $15.81 \%$. The vital signs such as rectal temperature, respiration rate, heart rate, and capillary refilling time were significantly $(p<0.01)$ increased in babesiosis positive dogs than healthy dogs (Table-1).

In this study, rectal temperature was elevated in only $5(31.25 \%)$ cases while remaining $(68.75 \%)$ of the dogs had near to normal temperature. Thus, it is apparent that pyrexia is not a cardinal sign in canine babesiosis due to $B$. gibsoni. These findings were in agreement with Varshney et al. [18] and Yadav et al. [19]. The respiration rate, heart rate, and capillary refilling time were increased than healthy dogs may be due to marked anemia and stress $[18,20]$.

\section{Clinical signs}

There were a wide variety of clinical signs were observed at the time of presentation including general state signs $(50.82 \%)$, gastrointestinal sings $(13.94 \%)$, cardiac signs $(13.11 \%)$, nervous signs $(13.11 \%)$, respiratory signs $(4.92 \%)$, and urological signs $(4.10 \%)$. The details of each clinical sign with percentage are presented in Table- 2 .

Here, it was seen that $50.82 \%$ of the dogs were having clinical signs of general state. The tick infestation was found in $93.15 \%(15 / 16)$ cases.

Godara et al. [21] stated that there was a positive correlation between the presence of ticks on the 
Table-1: Clinical variants (mean \pm SE values) in healthy and babesiosis positive dogs.

\begin{tabular}{lcc}
\hline Clinical variant & Healthy dogs & $\begin{array}{c}\text { Babesiosis } \\
\text { positive dogs }\end{array}$ \\
\hline Rectal temperature $\left({ }^{\circ} \mathrm{F}\right)$ & $102.00 \pm 00.21$ & $102.96 \pm 00.23^{* *}$ \\
Heart rate (beats/min) & $117.00 \pm 03.04$ & $129.88 \pm 02.94 * *$ \\
Respiration rate & $28.50 \pm 01.67$ & $36.56 \pm 02.22 * *$ \\
(breaths/min) & $01.25 \pm 00.16$ & $02.37 \pm 00.13^{* *}$ \\
CRT (seconds) & (s)
\end{tabular}

Table-2: Clinical signs in dogs with babesiosis.

\begin{tabular}{lc}
\hline Different forms & B. gibsoni $\mathbf{n = 1 6}(\mathbf{\%})$ \\
\hline General state signs & $15(93.15)$ \\
Ticks on body & $11(68.75)$ \\
Weakness/emaciation/apathy & $9(56.25)$ \\
Dehydration & $7(43.75)$ \\
Recumbancy/prostration & $3(18.75)$ \\
Petechiae/epistaxis/hemorrhage & $9(56.25)$ \\
Pale mucus membrane & $4(25.00)$ \\
Congested & $4(25.00)$ \\
Icteric & \\
Gastrointestinal signs & $8(50.00)$ \\
Anorexia & $4(25.00)$ \\
Vomiting/nausea & $2(12.50)$ \\
Diarrhea & $3(18.75)$ \\
Salivation & $11(68.75)$ \\
Nervous signs & $5(31.25)$ \\
Dullness/depression & $10(62.50)$ \\
Ataxia/paresis/epilepsy & $6(37.50)$ \\
Cardiac signs & \\
Arrhythmia & $3(18.75)$ \\
Weak pulse & $3(18.75)$ \\
Respiratory sign & \\
Nasal discharge/coughing & $3(18.75)$ \\
Dyspnea & $2(12.50)$ \\
Urological sign & \\
Dribbling of urine & \\
Hemoglobinuria & \\
\hline B. gibsoni=Babesia gibsoni & \\
\hline
\end{tabular}

B. gibsoni=Babesia gibsoni

body surface of host and hemoprotozoan infections. Weakness, dehydration, and recumbency were other non-specific clinical signs observed, accordance with Karunakaran et al. [22] and Yadav et al. [19]. Pale mucous membranes were due to marked anemia and petechiation was due to intravascular hemolysis. Jaundice is one of the most commonly reported complications of canine babesiosis [23]. Anemia is typically due to both intravascular and extravascular hemolysis [24]. Anorexia was evident in 50.0\% cases, and other similar, different gastrointestinal clinical findings in B. gibsoni positive dogs were also reported by Varshney et al. [18], Yadav et al. [19], and Kumar et al. [25]. Nervous system involvement was observed in $13.11 \%$ cases. Cerebral babesiosis occurs with the sludging of erythrocytes within central nervous system capillaries leading to tissue hypoxia, weakness, ataxia, seizures, and vestibular or cerebellar signs [26]. Cardiac arrhythmia and dyspnea may be to pulmonic changes or cardiopulmonary involvement [18]. Hemoglobinuria was observed only in two cases. Unlike bovine babesiosis, hemoglobinuria is rarely seen in canine babesiosis [18,27-29]. Oliguria is an ominous sign in dogs affected with renal impairment due to babesiosis [30]. These findings suggest that there is large variation in clinical signs in canine babesiosis and wide range of inconsistent clinical manifestations might be due to multisystemic effects of disease.

\section{Hematological parameters}

Levels of hemoglobin $(\mathrm{Hb})$, total erythrocyte count (TEC), and packed cell volume (PCV) decreased significantly in babesiosis positive dogs than healthy dogs (Table-3). Decrease in Hb levels were in agreement with reports of Furlanello et al. [31], Niwetpathomwat et al. [32], Selvaraj et al. [8], Shah et al. [33], Wadhwa et al. [34], Andoni et al. [35,36], Reddy et al. [37], and Nalubamba et al. [38].

Decreased TEC levels were in accordance with reports of Niwetpathomwat et al. [32], Shah et al. [33], Andoni et al. [35,36], Reddy et al. [37], and Nalubamba et al. [38]. Hb and TEC levels could be due to epistaxis, petechial hemorrhages, direct mechanical disruption caused by parasite as it leaves red blood cells, intravascular hemolysis, and immune-mediated or non-immune mediated destruction of red blood cells or due to severe anemia.

The difference between total leukocyte count and platelet count in dogs with babesiosis and healthy were statistically non-significant which was contrast to Shah et al. [33] and Sivajothi et al. [39] who reported significant decreased platelet counts (i.e., thrombocytopenia). Selvaraj et al. [8], Wadhwa et al. [34], and Reddy et al. [37] reported the presence of significant leukopenia in dogs with babesiosis. Decreased platelet counts in babesiosis were in agreement with reports of Furlanello et al. [31], Niwetpathomwat et al. [32], Shah et al. [33], Wadhwa et al. [34], Andoni et al. [35,36], Reddy et al. [37], Nalubamba et al. [38], and Vishnurahav et al. [40]. Babesia initiates a mechanism of antibody-mediated cytotoxic destruction of circulating erythrocytes and anemia may be more dependent on the host immune response than on the direct destruction of $\mathrm{RBC}$ by the piroplasm [41]. The mechanisms of the thrombocytopenia are not yet fully understood in babesiosis. Elevated body temperature could have contributory effect on thrombocytopenia [42]. Decreased PCV levels in dogs with babesiosis were in agreement with reports of Furlanello et al. [31], Niwetpathomwat et al. [32], Selvaraj et al. [8], Shah et al. [33], Wadhwa et al. [34], Andoni et al. [35,36], Reddy et al. [37], and Nalubamba et al. [38].

Among differential leukocyte count, levels of neutrophils increased significantly in dogs with babesiosis than healthy dogs. The reason might be due to coinfection. These findings were in accordance with reports of Selvaraj et al. [8], Shah et al. [33], and Vishnurahav et al. [40]. However, Reddy et al. [37] 
Table-3: Hematological parameters (mean $\pm \mathrm{SE})$ in healthy and babesiosis positive dogs.

\begin{tabular}{lcc}
\hline Parameter & $\begin{array}{c}\text { Healthy dogs } \\
(\mathbf{n = 8})\end{array}$ & $\begin{array}{c}\text { Babesiosis positive } \\
\text { dogs }(\mathbf{n = 1 6})\end{array}$ \\
\hline $\mathrm{Hb}(\mathrm{g} / \mathrm{dl})$ & $13.23 \pm 00.43$ & $09.33 \pm 00.80^{* *}$ \\
$\mathrm{TEC}\left(\times 10^{6} / \mu \mathrm{l}\right)$ & $06.66 \pm 00.30$ & $04.80 \pm 00.43^{* *}$ \\
$\mathrm{PCV}(\%)$ & $38.46 \pm 01.64$ & $30.26 \pm 02.62^{*}$ \\
TLC $\left(\times 10^{3} / \mu \mathrm{l}\right)$ & $12.30 \pm 00.91$ & $16.54 \pm 02.71$ \\
Neutrophils $(\%)$ & $66.62 \pm 01.60$ & $81.72 \pm 02.04 * *$ \\
Lymphocytes $(\%)$ & $29.00 \pm 00.75$ & $12.21 \pm 02.25 * *$ \\
Monocytes $(\%)$ & $02.87 \pm 00.54$ & $02.06 \pm 00.23$ \\
Eosinophils $(\%)$ & $01.50 \pm 00.32$ & $04.95 \pm 00.85^{* *}$ \\
Basophils $(\%)$ & $00.00 \pm 00.00$ & $00.00 \pm 00.00$ \\
Platelet count & $242.25 \pm 16.99$ & $246.59 \pm 35.68$ \\
$\left(\times 10^{3} / \mu \mathrm{l}\right)$ & & \\
MCV $(\mathrm{fl})$ & $64.64 \pm 01.60$ & $64.58 \pm 00.92$ \\
MCH $(\mathrm{pg})$ & $21.50 \pm 00.70$ & $22.60 \pm 01.08$ \\
MCHC $(\mathrm{g} / \mathrm{dl})$ & $33.05 \pm 00.56$ & $31.67 \pm 01.01$ \\
\hline
\end{tabular}

$* * \mathrm{p}<0.01, * \mathrm{p}<0.05$. TEC $=$ Total erythrocyte count, $\mathrm{PCV}=$ Packed cell volume, $\mathrm{TLC}=$ Total leukocyte count, $\mathrm{MCHC}=$ Mean corpuscular hemoglobin concentration, $\mathrm{MCV}=$ Mean corpuscular volume, $\mathrm{MCH}=$ Mean cell hemoglobin, $\mathrm{SE}=\mathrm{Standard}$ error, $\mathrm{Hb}=$ Hemoglobin

reported the decreased count of neutrophils in dogs with babesiosis. Levels of lymphocytes decreased significantly in dogs with babesiosis than healthy dogs. Lymphocytopenia may be due to concurrent viral infection associated with babesiosis. These findings were in accordance with reports of Selvaraj et al. [8] and Andoni et al. [35]. However, Shah et al. [33] and Reddy et al. [37] reported a significant increase in lymphocytes count in dogs with babesiosis. The differences between monocytes and basophils in dogs with babesiosis than healthy dogs were statistically non-significant. However, Reddy et al. [37] reported significant monocytes changes in dogs with babesiosis.

\section{Serum biochemical parameters}

Among various serum biochemical parameters, levels of alkaline phosphatase (ALP) increased significantly in dogs with babesiosis than healthy dogs (Table-4). These findings were in accordance with Shah et al. [33]. Increase in level of ALP was may be due to damage or abnormal function of biliary system [43]. Levels of alanine aminotransferase (ALT) increased significantly in dogs with babesiosis than healthy dogs. This present observation simulates to the findings of Wadhwa et al. [34] and Reddy et al. [37]. Levels of aspartate aminotransferase (AST) increased significantly in dogs with babesiosis than healthy dogs (Table-3). These findings were in agreement with Wadhwa et al. [34]. Increased activities of AST and ALT were might be due to escape of these enzymes from the damaged hepatic parenchymal cells with necrosis or altered membrane permeability indicating hepatic dysfunction [28]. Levels of total bilirubin increased significantly in dogs with babesiosis than healthy dogs. These findings were in accordance with Shah et al. [33]. Hyperbilirubinemia was due to resulted from both intravascular and extravascular hemolysis [44].
Table-4: Serum biochemical parameters (mean $\pm \mathrm{SE})$ in healthy and babesiosis positive dogs.

\begin{tabular}{lcc}
\hline Parameter & $\begin{array}{c}\text { Healthy dogs } \\
(\mathbf{n = 8})\end{array}$ & $\begin{array}{c}\text { Babesiosis } \\
\text { positive dogs } \\
(\mathbf{n = 1 6 )}\end{array}$ \\
\hline ALP (IU/L) & $78.38 \pm 04.15$ & $283.06 \pm 30.81^{* *}$ \\
ALT (IU/L) & $34.81 \pm 03.20$ & $69.06 \pm 05.80^{* *}$ \\
AST (IU/L) & $45.44 \pm 04.83$ & $67.55 \pm 03.77^{* *}$ \\
Total bilirubin (mg/dl) & $00.40 \pm 00.05$ & $00.67 \pm 00.08 *$ \\
BUN (mg/dl) & $14.84 \pm 00.88$ & $29.96 \pm 02.50$ \\
Creatinine (mg/dl) & $01.03 \pm 00.12$ & $00.93 \pm 00.10$ \\
Total protein (g/dl) & $05.90 \pm 00.37$ & $05.46 \pm 00.46$ \\
Albumin $(\mathrm{g} / \mathrm{dl})$ & $01.72 \pm 000.14$ & $02.37 \pm 00.20$ \\
Globulin $(\mathrm{g} / \mathrm{dl})$ & $01.47 \pm 00.24$ & $03.23 \pm 00.38$ \\
A:G $(\mathrm{g} / \mathrm{dl})$ & $01.29 \pm 00.15$ & $01.37 \pm 00.08$ \\
\hline
\end{tabular}

$* * p<0.01, * p<0.05$. ALP=Alkaline phosphatase, ALT=Alanine aminotransferase, AST=Aspartate aminotransferase, BUN=Blood urea nitrogen, $\mathrm{SE}=$ Standard error

Levels of blood urea nitrogen (BUN) increased significantly in dogs with babesiosis than healthy dogs. Reddy et al. [37] also recorded significant increase in level of BUN in babesiosis positive dogs and healthy dogs. Here, only the level of BUN is increased indicative of non-renal influences. This elevation may be due to hemolysis of RBCs, gastrointestinal hemorrhage or blood transfusion. Elevations in serum urea may be caused by a recent protein meal or increased tubular reabsorption during low tubular urine flow rates. A recent protein meal, however, is an unlikely event in canine babesiosis patients because of appetite depression [45]. The difference between values of creatinine in babesiosis positive dogs and healthy dogs were statistically nonsignificant. These findings were in agreement with Wadhwa et al. [34]. However, Andoni et al. [36], Reddy et al. [37], and Vishnurahav et al. [40] reported significant increase in creatinine in babesiosis positive dogs and healthy dogs.

Levels of albumin and $A: G$ ratio decreased significantly $(p<0.01)$ in dogs with babesiosis than healthy dogs. These results were in accordance with Yadav et al. [19]. Levels of globulin increased significantly $(\mathrm{p}<0.01)$ in dogs with babesiosis than healthy dogs. These finding were in agreement with Vishnurahav et al. [40] and Vijayalakshmi et al. [46]. The difference between values of TPP in babesiosis positive dogs and healthy dogs were statistically nonsignificant. These findings were in accordance with Reddy et al. [37].

\section{Conclusion}

The overall prevalence of babesiosis, based on peripheral blood smear examination was recorded as $20.25 \%$. The dogs with naturally occurring babesiosis exhibited clinical signs include tick infestation, emaciation, anorexia, and dullness. Characteristic hematological pattern associated with babesiosis in dogs showed that there was decreased level of $\mathrm{Hb}$, TEC, and PCV as well as increased level of neutrophils while serum biochemical pattern include 
increased the level of ALP, ALT, AST, total bilirubin, and globulin. During the study, it was observed that $B$. gibsoni had a wide variety of clinical manifestations so dogs are showing erratic fever, weight loss, depression, pale mucosa, and splenomegaly alone or in combination can be suspected for babesiosis. All the suspected dogs in this study were diagnosed only based on the microscopic examination of the stained peripheral blood smears. Microscopic examination may not detect low parasitemia though; it remains the most rapid confirmatory method which was carried out in this study. As the disease is spread through vector tick, control of the vector tick is essential for prevention of disease. Application of ectoparasiticides with acaricidal/insecticidal and additional repellent efficacy reduces the arthropod-host interaction including attachment to the skin and blood feeding and can thus reduce the risk of infection. Prevention of tick attachment and flea or sand fly or mosquito bites must be an established tool of disease prophylaxis in any dog living in vector endemic areas or traveling with its owner to such regions. Dog owners should be made aware of the risks and the need for protection by their veterinarians.

\section{Authors' Contributions}

The present study was a part of AKB's original research work which includes experimental design, a collection of blood and serum samples, examination of blood smear, estimation of hematobiochemical parameters and statistical analysis, preparing, and drafting the manuscript during M.V.Sc. thesis program. GCM had designed the plan of work as well as provided guidance during the entire experiment and corrected manuscript. FBT helped during sampling, statistical analysis, and manuscript preparation. All authors read and approved the final manuscript.

\section{Acknowledgments}

This study was funded by College of Veterinary Science and Animal Husbandry, Anand Agricultural University, Anand, Gujarat. Authors are thankful to the staff of TVCC, Nandini Veterinary Hospital, Surat and the Department of Veterinary Physiology and Biochemistry at College of Veterinary Science and Animal Husbandry, Anand Agricultural University, Anand, Gujarat, as well as dog owners of different areas for their valuable cooperation.

\section{Competing Interests}

The authors declare that they have no competing interests.

\section{References}

1. Companion Vector-Borne Diseases (CVBD). (2017) $29^{\text {th }}$ August. Available from: http://www.cvbd.org. Accessed on 2017-08-29.

2. Bruno, C. (2011) Tick-borne infections in dogs: An emerging infectious threat. Vet. Parsitol., 197: 294-301.

3. Okubanjo, O.O., Adeshina, O.A., Jatau, I.D. and Natala, A.J. (2013) Prevalence of Babesia canis and Hepatozoon canis in Zaria, Nigeria. Sokoto J. Vet. Sci., 11(2): 15-20.

4. Irwin, P.J. (2010) Canine babesiosis. Vet. Clin. Small Anim., 40: 1141-1156.

5. Taboada, J. and Merchant, S.R. (1991) Babesiosis of companion animals and man. Vet. Clin. North Am. Small Anim. Pract., 21: 103-123.

6. Kjemtrup, A.M. and Conrad, P.A. (2006) A review of the small canine piroplasms from California: Babesia conradae in the literature. Vet. Parasitol., 138: 112-117.

7. Abd Rani, P.A.M., Irwin, P.J., Coleman, G.T., Gante, M. and Traub, R.J. (2011) A survey of canine tick-borne diseases in India. Parasit. Vectors, 4: 141.

8. Selvaraj, P., Kumar, K.S., Vairamuthu, S., Prathaban, S. and Srinivasan, S.R. (2010) Babesia gibsoni - An emerging challenge in canine pediatric practice in Chennai. Tamilnadu. J. Vet. Anim. Sci., 6(3): 122-124.

9. Singh, H., Singh, N.K. and Rath, S.S. (2012) A survey of canine babesiosis in and around Ludhiana district, Punjab. Indian J. Canine Pract., 4: 2.

10. Jadhav, K.M. and Ambegaonkargupte, R.U. (2015) Studies on Epidemiology of Canine Babesiosis in Gujarat. In: XXXIII - ISVM Annual Convention and National Symposium on New Dimensions in Veterinary Medicine: Technological Advances, One Health Concept and Animal Welfare Concerns at Pookode, Kerala, $22^{\text {nd }}-24^{\text {th }}$ January. p45.

11. Uilenbersg, G. (2006) Babesia - A historical overview. Vet. Parasitol., 138(1-2): 3-10.

12. Aysul, N., Ural, K., Ulutas, B., Eren, H. and Karagenc, T. (2013) First detection and molecular identification of Babesia gibsoni in two dogs from the Aydin Province of Turkey. Turk. J. Vet. Anim. Sci., 37: 226-229.

13. Jefferies, R., Ryan, U.M., Jardine, J., Broughton, D.K., Robertson, I.D. and Irwin, P.J. (2007) Blood, bull terriers and babesiosis. Further evidence for direct transmission of Babesia gibsoni in dogs. Aust. Vet. J., 85: 459-460.

14. Pedersen, N.C. (1999) A review of immunologic diseases of the dog. Vet. Immunol. Immunopathol., 69: 251-342.

15. Irwin, P.J. (2005), Babesiosis and cytauxzoonosis. In: Shaw, S.E., Day, M.J., editors. Arthropod-borne Infectious Diseases of the Dog and Cat. Manson Publishing, Barcelona. p63-77.

16. Irwin, P.J. and Hutchinson, G.W. (1991) Clinical and pathological findings of Babesia infection in dogs. Aust. Vet. J., 68: 204-209.

17. Snedecor, G.W. and Cochran, W.G. (1990) Statistical Methods. $6^{\text {th }}$ ed. Oxford and JBH Publishing, New York.

18. Varshney, J.P., Deshmukh, V.V. and Chaudhary, P.S. (2008) Multisystem effects of canine babesiosis and management of critical cases. Intas Polivet, 9(II): 281-287.

19. Yadav, R., Gattani, A., Gupta, S.R. and Sharma, C.S. (2011) Jaundice in dog associated with babesiosis - A case report. Int. J. Agro Vet. Med. Sci., 5(1): 3-6.

20. Deshmukh, V.V., Varshney, J.P. and Chaudhary, P.S. (2008) Management of respiratory syndrome associated with a mixed infection of Babesia canis, Babesia gibsoni and Ehrlichia canis in a Great Dane Pup. Intas Polivet, 9(II): 210-212.

21. Godara, R., Sharma, R.L., Sharma, C.S. and Sharma, D.K. (2010) Parasitic infections in dogs in semi-arid Jaipur (Rajasthan). J. Vet. Parasitol., 24: 83-86.

22. Karunakaran, S., Pillai, U.N. and Sasidharan, H.P. (2011) Babesia gibsoni infection in a German Shepherd dog. Vet. World, 4(6): 269-270.

23. Lobetti, R.G. (1998) Canine babesiosis. Compendium on continuing education for the practicing veterinarian.20: 418-431.

24. Boozer, L. and Macintire, D. (2005) Babesia gibsoni: An emerging pathogen in dogs. Compendium, 2: 33-42.

25. Kumar, V., Pal, H. and Sharma, H. (2015) Canine Babesiosis - A case report. Ann. Vet. Anim. Sci., 2: 1.

26. Suresh, R.V., Prasad, A.A., Prathaban, S., Simon, M.S. and 
Balachandran, C. (2010) Cerebral babesiosis in a dog - A case report. Indian. J. Field Vet. 5: 31-32.

27. Saud, N., Hazarilka, G.C., Chakravorti, P. and Rajkhowa, S. (2000) Clinico-haematological findings of Canine babesiosis. Indian Vet. J., 77: 1034-1036.

28. Gupta, S.K., Bhardwaj, R.K., Singh, R., Raina, A.K. and Avinash, K. (2002) Management of canine babesiosis - A case report. Indian J. Vet. Med., 22: 124.

29. Varshney, J.P., Varshney, V.P. and Hoque, M. (2003) Clinicohaematological, biochemical, endocrinological and ultrasonography findings in canine babesiosis. Indian $J$. Anim. Sci., 73: 1099-1101.

30. Lobetti R.G., Jacobson, L.S. (2001) Renal involvement in dogs with babesiosis. J. S. Afr. Vet. Assoc., 72: 23-28.

31. Furlanello, T., Fiorioa, F., Caldina, M., Lubasb, G. and Solano-Gallegoa, L. (2005) Clinicopathological findings in naturally occurring cases of babesiosis caused by large form Babesia from dogs of northeastern Italy. Vet. Parasitol., 134: 77-85.

32. Niwetpathomwat, A., Somporn, L., Suvarnavibhaja, S. and Assarasakorn, S. (2006) A retrospective study of clinical hematology and biochemistry of canine babesiosis on hospital populations in Bangkok, Thailand. Comp. Clin. Pathol., 15(2): 110-112.

33. Shah, S.A., Sood, N. and Tumati, S.R. (2011) Haematobiochemical changes in natural cases of canine babesiosis. Asian J. Anim. Sci., 5(6): 387-392.

34. Wadhwa, D.R., Pal, B., Mandial, R.K., Kumar, A. and Agnihotri, R.K. (2011) Clinical, haemato-biochemical and therapeutic studies of canine Babesiosis in Kangra valley of Himachal Pradesh. J. Vet. Parasitol., 25(1): 39-41.

35. Andoni, E., Rapti, D., Postoli, R. and Zalla, P. (2012) Hematologic changes in dogs naturally infected with Babesia. Albanian J. Agric. Sci., 11: 3.

36. Andoni, E., Rapti, D., Postoli, R., Dimco, E. and Abeshi, J. (2013) Clinicopathological findings in dogs naturally infected with Babesia spp. Albanian J. Agric. Sci., 12(2): 185-189.
37. Reddy, B.S., Sivajothi, S., Reddy, L.S.S. and Raju, K.G.S. (2014) Clinical and laboratory findings of Babesia infection in dogs. J. Parasit. Dis. 92: 268-272.

38. Nalubamba, K.S., Mudenda, N.B., Namwila, M.M., Mulenga, C.S., Bwalya, E.C., Kandawire, E.M., Saasa, N., Hancanga, C., Oparaocha, E. and Simuunza, M. (2015) A study of naturally acquired canine babesiosis caused by single and mixed Babesia species in Zambia: Clinicopathological findings and case management. J. Parasitol. Res., 9: 236-249.

39. Sivajothi, S., Reddy, B.S., Rayulu, V.C. and Venkatasivakumar, R. (2014) Babesiosis in dogs: A report of two different cases. Adv. Appl. Sci. Res., 5(3): 276-279.

40. Vishnurahav, R.B., Pillai, U.N., Alex, P.C., Ajitkumar, S. and Lusy, S. (2014) Haemato-biochemical changes in canine babesiosis. Indian J. Canine Pract., 6: 2.

41. Boozer, A.L. and Macintire, D.K. (2003) Canine babesiosis. Vet. Clin. North Am. Small Anim. Pract., 33: 885-904.

42. Oglesbee, M.J., Diehl, K., Crawford, E., Kearns, R. and Krakowka, S. (1999) Whole body hyperthermia: Effects upon canine immune and hemostatic functions. Vet. Immunol. Immunopathol., 69: 185-199.

43. Crnogaj, M., Petlevski, R., Mrljak, V., Kis, I., Torti, M., Kucer, N., Matijatko, V., Sacer, I. and Stokovic, I. (2010) Malondialdehyde levels in serum of dogs infected with Babesia canis. Vet. Med., 55(4): 163-171.

44. Irwin, P.J. and Hutchinson, G.W. (1991) Clinical and pathological findings of Babesia infection in dogs. Aust. Vet. J., 68: 204-209.

45. Scally, M.P., Lobetti, R.G., Reyers, F. and Humphris, D. (2004) Are urea and creatinine values reliable indicators of azotaemia in canine babesiosis? Tydskr. S. Afr. Vet. Ver., 75(3): 121-124.

46. Vijayalakshmi, P., Srinivasan, S.R., Vairamuthu, S., Mangalagowri, A., Latha, B.R. and Nambi, A.P. (2014) Clinico pathological features in dogs associated with babesiosis. Ind. Vet. J., 91(4): 21-22. 